\title{
Hipparcos Data Validation
}

\author{
Floor van Leeuwen \\ Institute of Astronomy, Madingley Road, Cambridge, UK
}

\begin{abstract}
An overview is presented of tests performed to check the statistical properties of the formal errors on the Hipparcos parallaxes. It is shown that there is no evidence for systematic or correlated errors beyond a correlation level of 0.12 and an angular scale of 1.2 degrees.
\end{abstract}

\section{Introduction}

The formal errors on the Hipparcos parallaxes are of importance for any study involving stars for which the signal to noise ratio of the measured parallax is small (less than 5). Aspects of using parallaxes with lower signal to noise ratios have been discussed by Brown et al. (1997). The general rule in all these cases is to interpret the data in parallax space. Two more aspects of importance when interpreting low signal-to-noise ratio parallax data are the relation between the formal and actual error distributions and the correlations between errors on parallaxes of stars at small separations on the sky. The latter question is in particular of importance for the derivation of open cluster parallaxes (van Leeuwen \& Evans 1998).

\section{The formal errors on the Hipparcos parallaxes}

The Hipparcos parallaxes have been obtained from the FAST and NDAC data reductions, in a complicated process which involved an assessment of the formal errors on the abscissa measurements as a function of magnitude, and of the correlations between the abscissa errors as produced by FAST and NDAC. For a detailed discussion the reader is referred to ESA (1997), Volume 3, Chapter 17. The distribution of the abscissa measurements and their formal errors determined the formal errors on the measured parallaxes. These were tested in at least two ways. Lindegren (1995) compared the observed tail of negative parallaxes in the parallax distribution with the expected tail given the formal errors, and concluded that, statistically, the formal errors are within 1 per cent of the actual errors. Arenou et al. (1995) examined the distribution of parallaxes for objects with external parallax estimates well below the measuring accuracy of Hipparcos (for example stars in the LMC and SMC), obtaining very similar results. The importance of these findings is that there are no significant unaccounted for errors present in the Hipparcos parallaxes, contrary to claims made by e.g. Narayanan \& Gould (1999), as such errors would have shown up in the statistical tests. 


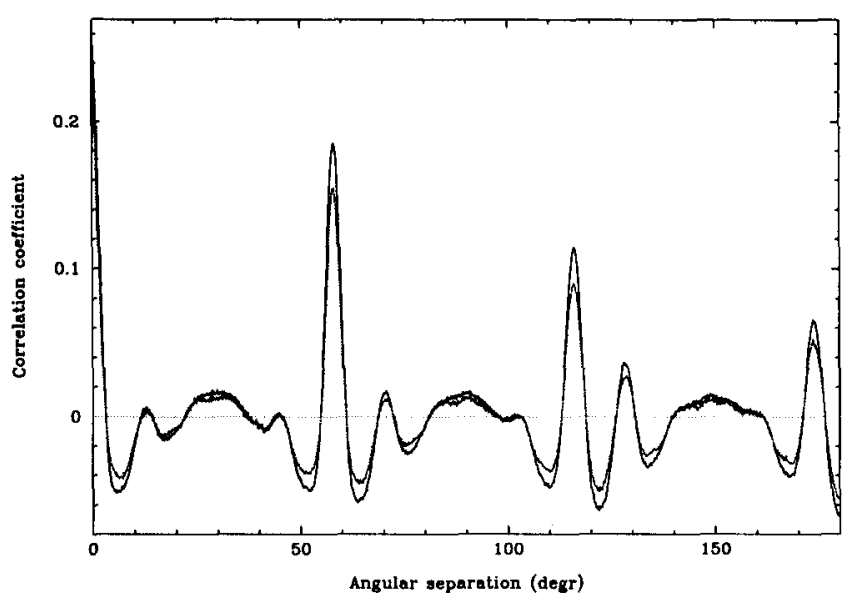

Figure 1. The abscissa residuals correlation coefficient as a function of the separation on the reference great circle for all data sets. The NDAC curve can be distinguished from the FAST curve by its lower minima and higher maxima, differences that become more pronounced as data sets become shorter.

\section{Correlated errors in the Hipparcos data}

When combining Hipparcos parallax data for a group of stars, like using the members of an open cluster to determine its distance, the correlations between the errors on the parallaxes of the individual members have to be accounted for. It had been known from before the launch of Hipparcos that some degree of correlation would exist between the astrometric data of neighbouring stars (Lindegren 1989). These correlations are the result of correlated errors on the underlying abscissa determinations, which were caused by the requirement to estimate from the measured transit times both the corrections to the along-scan phase and to the individual stellar abscissae. The abscissa data for the final mission product have been published on a CDRom, included in Vol.17 of ESA (1997), and allowed an investigation of the actual levels of the correlations. This was done by Van Leeuwen (1997) (see also van Leeuwen \& Evans 1998) and van Leeuwen (1999a, 1999b, 2000). Fig. 1 shows the correlation coefficients between the abscissa residuals for all mission data. Examining subgroups defined by the length of the observation period used to build up the great circle shows that correlations tend to be higher for short sets, and lower for long sets, with a maximum of 0.35 to 0.4 , and an average of 0.2 at close to zero separation.

An important aspect in the relation between the abscissa correlations and correlations between the final astrometric parameters for neighbouring stars is the fraction of great circles shared between those neighbouring stars. This function was derived using 20 randomly chosen stars, comparing the great circles used for each with those of all stars in a radius of $7^{\circ} .3$ around them. Fig. 2 shows the averaged results: at a separation of only $1^{\circ}$ the coincidence fraction has dropped to 50 per cent, implying that for larger separations, more than half the data has been obtained completely independently. This considerably reduces 


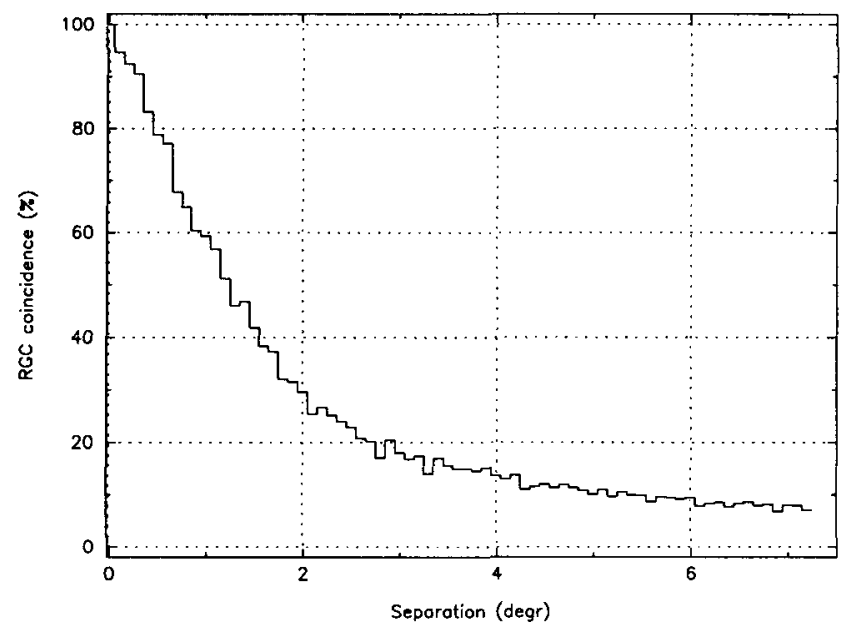

Figure 2. The coincidence of RGCs between a target star and all Hipparcos stars in a 7.3 radius around it. The histogram shows the averaged results for 20 randomly chosen target stars as a function of distance from the target star.

the penetration of the abscissa correlations into the astrometric parameters. It also shows that one cannot expect any correlations to exist between parallax errors at scales of $2^{\circ}$ to $3^{\circ}$ as was claimed by Narayanan \& Gould (1999).

A test on the correlation coefficient of the errors on the parallaxes of neighbouring stars is possible thanks to the work by Dravins et al. (1997) and Madsen (1999) on the Hyades cluster. In the case of the Hyades stars, kinematic parallaxes can be derived for individual stars, using the proper motion and position on the sky, and identical space velocities for all cluster members (this is true apart from the internal velocity dispersion, which is small for the Hyades stars). These kinematic parallaxes are of a higher precision than the directly measured parallaxes. An examination of their differences can therefore reveal correlations that may exist between the errors on the directly measured parallaxes. This was done by means of an expression describing the correlations as a function of separation on the sky. The function was given by a simple parabola, with its maximum at zero separation, and correlations were assumed to be zero beyond the separation where the parabolic function reached zero. This gave two parameters to optimize: the correlations at zero separation, and the correlation cut-off radius. Using a grid of these parameters, each time decorrelating the residuals, the standard deviation of the decorrelated and weighted residuals was minimized (see Fig. 3 in van Leeuwen 2000). The optimal values found were 1.2 degrees for the cut-off radius, and 0.114 for the maximum correlation. These values are in very good agreement with what should be expected from the build-up of the abscissae correlations and the great circle coincidence fraction as described above. There are no signs of other, larger scale correlations, contrary to claims made by Narayanan \& Gould (1999). The methods outlined by van Leeuwen \& Evans (1998), and used by Robichon et al. (1999) and van Leeuwen (1999) for the 
determination of cluster parallaxes, take into account the correlations between the abscissa errors, and derive single solutions for the astrometric parameters of open clusters from the decorrelated abscissa measurements of the individual cluster members. The main effect of this is that the formal error on the cluster parallax is properly calculated.

\section{Conclusions}

Though there are claims in the literature of systematic errors in the Hipparcos parallaxes (only in order to explain an unexpected result for the Pleiades cluster), there is no statistical evidence in the data itself. In as far as the Pleiades cluster is concerned, there is no model-independent determination of the Pleiades parallax that deviates significantly from the Hipparcos determination, though the ground based observations seem to be in surprisingly good agreement with each other given the formal errors quoted. To use the lack of agreement with theoretical models as an indicator of errors in the data (Pinsonneault et al. 1998) seems like turning astronomy upside down.

\section{References}

Arenou, F., Lindegren, L., Froeschlé, M., Goméz, A. E., Turon, C., Perryman, M. A. C., \& Wielen, R. 1995, A\&A, 304, 52

Brown, A.G.A., Arenou, F., van Leeuwen, F., Lindegren, L., \& Luri, X. 1997, ESA SP-402, 63

Dravins, D., Lindegren, L., Madsen, S., \& Holmberg, J. 1997, ESA SP-402, 733 ESA 1997, The Hipparcos and Tycho catalogues, ESA SP-1200

van Leeuwen, F. 1997, ESA SP-402, 203

van Leeuwen, F. 1999a, A\&A, 341, L71

van Leeuwen, F. 1999b, in ASP Conf. Ser. Vol. 167, Harmonising Cosmic Distance Scales in a Post-Hipparcos Era, ed. D. Egret \& A. Heck (San Francisco: ASP), 52

van Leeuwen, F. 2000, in ASP Conf. Ser. Vol. 198, Stellar Clusters and Associations: Convection, Rotation and Dynamos, ed. R. Pallavicini, G. Micela, \& S. Sciortino (San Francisco: ASP), 85

van Leeuwen, F., \& Evans, D.W. 1998, A\&AS, 130, 157

Lindegren, L. 1989, ESA SP-1111, Vol.III, 311

Lindegren, L. 1995, A\&A, 304, 61

Madsen, S. 1999, in ASP Conf. Ser. Vol. 167, Harmonising Cosmic Distance Scales in a Post-Hipparcos Era, ed. D. Egret \& A. Heck (San Francisco: ASP), 78

Narayanan, V.K., \& Gould, A. 1999, ApJ, 523, 328

Pinsonneault, M.H., Stauffer, J., Soderblom, D. R., King, J. R., \& Hanson, R. B. 1998, ApJ, 504, 170

Robichon, N., Arenou, F., Mermilliod, J.-C., \& Turon, C. 1999, A\&A, 345, 471 